\title{
Supercontinuum generation in nanostructured core gradient index fibers
}

\author{
Xavier Forestier ${ }^{1,2} \cdot$ Tanvi Karpate $^{1,2} \cdot$ Guillaume Huss $^{3} \cdot$ Vincent Tombelaine $^{3} \cdot$ Grzegorz Stępniewski $^{1}$. \\ Alicja Anuszkiewicz ${ }^{1,4} \cdot$ Rafał Kasztelanic $^{1,2} \cdot$ Adam Filipkowski $^{1,2} \cdot$ Dariusz Pysz $^{1} \cdot$ Mariusz Klimczak $^{2}$. \\ Ryszard Buczyński ${ }^{1,2}$
}

Received: 31 December 2019 / Accepted: 22 February 2020 / Published online: 10 March 2020

(c) The Author(s) 2020

\begin{abstract}
We report on near-infrared supercontinuum generation in a submeter-long single-mode, nanostructured core fiber. The fiber core is composed of few thousand pure silica and germanium-doped silica glass nanorods with diameter of $200 \mathrm{~nm}$ each. The nanorods' distribution is calculated based on the Maxwell-Garnett effective medium approach to mimic effective parabolic refractive index distribution in the fiber core. The standard stack-and-draw method was used to scale down the fiber structure and obtain subwavelength nanorods in the core. Size and distribution of individual nanorods are essential to determine modal and dispersion properties of the fiber without assistance of air holes in the fiber cladding. We study supercontinuum generation performance in this nanostructured core fiber pumping with low-cost microchip laser operating at $1550 \mathrm{~nm}$ with $1 \mathrm{~ns}$ pulse length and pulse energy of $0.4 \mu \mathrm{J}$. A modulation instability-driven supercontinuum is generated in the fiber, covering a wavelength span of 1400-2300 nm. Due to possibility of dispersion engineering and all-solid structure the nanostructured fibers offer new possibilities for development of low-cost all-fiber supercontinuum light sources for the near-infrared range and cascaded ultrabroadband supercontinuum all-fiber systems.
\end{abstract}

Keywords Nanostructured optical fibers $\cdot$ Graded-index optics $\cdot$ Supercontinuum generation $\cdot$ Nonlinear optics

\section{Introduction}

Since its introduction nearly 2 decades ago, optical fiberbased supercontinuum has become the light source of first choice in research as well as for industrial applications in optical coherent tomography (Fujimoto 2003), frequency combs generation for high precision metrology (Jones et al. 2000), and in spectroscopy, LIDAR, biomedical imaging and microscopy (Shi et al. 2004). Future application-specific requirements, such as small-form factor, high-intensity

Ryszard Buczyński

rbuczyns@igf.fuw.edu.pl

1 Eukasiewicz - Institute of Electronic Materials Technology, Wolczynska 133, 01-919 Warsaw, Poland

2 University of Warsaw, Faculty of Physics, Pasteura 5, 02-093 Warsaw, Poland

3 LEUKOS, 37 rue Henri Giffard, 87280 Limoges, France

4 Institute of Electronic Systems, Warsaw University of Technology, Nowowiejska 15/19, 00-665 Warsaw, Poland coverage of a specific wavelength range or beam shape can still be addressed with dedicated optical fiber designs. Optical fibers have been the primary medium for supercontinuum generation for their strong confinement of pump laser light, as well as for the feasibility of chromatic dispersion engineering, which significantly simplifies matching of the nonlinear medium's optical properties to intended pump lasers, compared to non-fiber systems (Alfano and Shapiro 1970a, b; Dudley et al. 2006). Coherent and spectral properties of fiber-based supercontinuum can be widely modified to fulfill various application requirements (Klimczak et al. 2017; Wetzel et al. 2018; Genier et al. 2019). In 2000, Ranka et al. (2000) demonstrated for the first time supercontinuum generation in photonic crystal fibers (PCFs). Use of PCFs for supercontinuum generation proved a disruptive step forward in the field, because relevant characteristics of a PCF, most notably chromatic dispersion profile, can be modified by the PCF lattice geometry (Dudley et al. 2006). PCFs have been reported with various designs, such as hexagonal lattice structure, suspended core structure, wherein they can be engineered for their properties such as dispersion, 
confinement losses, effective mode areas, and nonlinearity. This enabled improved efficiency and control of supercontinuum generation and its dynamics than in classic, stepindex fibers. Supercontinuum generation in PCFs has been intensively studied leading covering wavelengths from the ultraviolet (Jiang et al. 2015), the visible up to the midinfrared (Wei et al. 2005; Liu et al. 2016). In this context, different glasses are under investigation to target different wavelength ranges and properties. The most common and industry-accepted standard for optical fiber development, including PCF drawing, is the silica glass due to its stable mechanical and chemical properties, the telecommunication systems applications and bio-inert character, making it the glass of choice for biomedical sensing applications (Śmietana et al. 2016). For supercontinuum generation in the mid-infrared wavelengths, various soft glasses such as tellurite, fluoride or chalcogenide are considered for "classic" step-index fibers or index guiding PCFs, as these glasses have broad transmission windows exceeding that of silica.

The most efficient way to maximize spectral width in supercontinuum generation relies on high power pumping into anomalous dispersion, close to a zero-dispersion wavelength in a positive-slope chromatic dispersion PCFs (Ranka et al. 2000). If a nanosecond pulsed laser is used (e.g., a Q-switched microchip laser), the physical mechanism constituting spectral broadening is noise amplification or modulation instability (Bandelow and Demircan 2005). This enables highly robust and cheap supercontinuum lasers covering wavelengths from the visible to the long-wave near-infrared and has been widely accepted by vendors and industry (Leukos; NKTPhotonics). Extension of spectral coverage beyond near-infrared is also possible in cascaded fiber supercontinuum setups, involving use of different glass fibers with matched mode areas-such devices have been shown as important for e.g., microelectronics industry OCT imaging (Israelsen et al. 2019). Owing to the fiber form of the nonlinear medium and the guided mode nature of the supercontinuum pulses, spatial coherence of the driving laser is preserved during the nonlinear broadening process, although temporal coherence is not. Temporal decoherence can be avoided at the cost of using a femtosecond driving laser, instead of a low-cost nanosecond laser (Dudley and Coen 2002).

Progress in development of high pulse energy, allfiber oscillators, as well as fiber concatenating technology prompted introduction of interesting all-fiber system for supercontinuum light sources (Nicholson et al. 2003; Chen et al. 2011; Petersen et al. 2016; Hudson et al. 2017; Tarnowski et al. 2019). All-fiber supercontinuum systems are highly advantageous as they are robust, compact and lack the need for alignment, which makes them attractive for use by non-specialists. In this case, use of PCFs brings a drawback related to their air-glass microstructure.
Open air holes in the PCF photonic cladding can accumulate humidity from environment, which can influence chromatic dispersion and confinement loss of fibers, as well as their reliability. The parameters of the air holes are changed during fusion splicing in proximity of the fiber interconnects and thus the procedure can be detrimental for confinement (i.e., increase confinement loss) or lead to excitation of unintended polarization mode components in polarization maintaining PCFs.

To overcome problems related to air-glass structure of photonic crystal fibers an all-glass photonic crystal fibers were introduced (Feng et al. 2003; Liu et al. 2016; Klimczak et al. 2016). The combined necessities of thermally matching the constituent glasses and assuring reasonable degree of chromatic dispersion engineering flexibility practically ruled out use of the industry-accepted silica glass in these works. Consequently, such fibers suffer from high attenuation and are almost impossible to fusion splice with standard silica fibers.

Recently, we have introduced the concept of all-glass nanostructured core fibers as a robust solution for industrial applications (Anuszkiewicz et al. 2018). In this case, arbitrary refractive index distribution in the fiber can be obtained (Michalik et al. 2019). Graded-index fiber, commonly used methods as Modified Chemical Vapor Deposition (MCVD) or ion exchange techniques (Oh 2012) allow only to obtain optical fibers with simple gradient index profiles with rotational symmetry. They do not allow the use of complex multicomponent glasses or development of arbitrary refractive index profile graded-index core fibers.

In the nanostructured core fibers modal, polarization and dispersion properties of the fibers are determined by internal nanostructure in the fiber core without the need of contribution from the air-glass cladding geometry. Recently, a new type of structuration demonstrated promising possibilities for fabrication of nanostructured graded-index (nGRIN) fibers with arbitrarily shaped index profiles (Hudelist et al. 2009; Siwicki et al. 2017; Kasztelanic et al. 2018). It consists in the creation of a transverse binary pattern of two materials with different refractive indices, with each element of it being subwavelength in diameter in the final fiber to obtain an average refractive index profile across the core area, according to the effective medium theory (EMT).

This new method opens up considerable versatility for chromatic dispersion, index distribution profile, polarization and modal engineering. In the work Michalik et al. (2019), we recently demonstrated an effective way of controlling the properties of the hybrid fibers by dispersion management. This serves the same advantage as that of PCFs provided with air hole microstructure engineering. Other advantages include the feasibility of development of multicore fibers and large-mode area (LMA) fibers, which are important for power scaling of lasers. Indeed, LMA fibers developed 
using the nGRIN approach have been reported in our previous works (Franczyk et al. 2018; 2019).

The nano-structuration of all-solid fiber also provides a clear mechanical advantage compared to 'holey' fibers (PCFs) and in addition to that, it makes fibers less prone to energy-related damages, due to its "volume glass" nature, compared to air-glass lattice PCFs. We recently introduced and validated experimentally the concept of silica-based single-mode nGRIN fiber (Anuszkiewicz et al. 2018). Using numerical simulations, we further demonstrated with a theoretical concept for a potential mid-IR gradient index fiber (Siwicki et al. 2017). We also validated that this extremely complex structure does not suffer from internal stresses due to the stacking process and that the loss of the fiber is comparable to that of commercial telecom fibers (Anuszkiewicz et al. 2019).

In this work, we validate the use nanostructured core graded-index all-solid fiber for near-infrared supercontinuum generation. We use silica-based nGRIN fiber with parabolic profile to verify how internal nanostructure influences supercontinuum generation. Besides a series of prototype nGRIN fibers for experiments, we used only industry-accepted components, such as commercial supercontinuum pump lasers to establish basis for potential new all-fiber-based supercontinuum source. In particular, we use a standard, low-cost $1 \mathrm{~ns}$ pulsed laser diode operating around $1550 \mathrm{~nm}$ as the pump

(a)

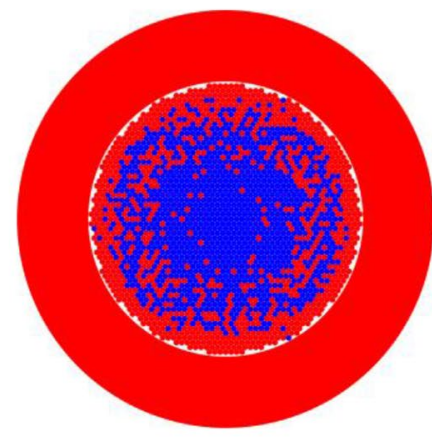

(b)

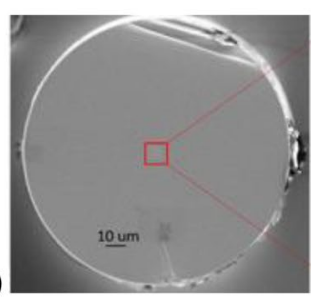

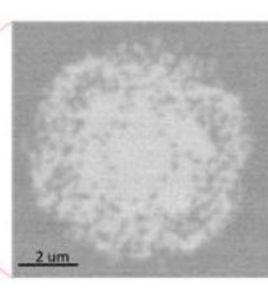

Fig. 1 a Core structure of the developed nGRIN fiber with an effective parabolic index profile. The core is composed of 2107 glass rods of low index fused silica (red) and high index $\mathrm{GeO}_{2}$-doped silica (blue). b Material dispersion of the two glasses used to form nGRIN source. We begin with a brief description of the fiber used, including its design, fabrication and linear, as well as nonlinear characteristics. Next, we present experimental results on supercontinuum generation supported with contributing phenomena explanation based on numerical modeling results obtained using nonlinear Schrödinger equation.

\section{Materials and methods}

\section{Fiber design and development}

The nanostructured core fiber preform was manually prepared by the classic stack-and-draw method, in a hexagonal lattice, according to the pattern shown in Fig. 1a. The core was composed of 2107 glass rods of two different materials. Two thermally matched silica-based glasses with different refractive indices were selected. Commercial fused silica glass Ohara SK1310 glass, with refractive index of $n_{\mathrm{d}}=1.4609$ at $546 \mathrm{~nm}$, was used as the low refractive index material of the core. Germanium oxide doped silica glass (containing $4.9 \mathrm{~mol} \% \mathrm{GeO}_{2}$ ) from OptaCore was selected as high refractive index core material. F300 fused silica tube from Heraeus was used to form the fiber cladding. Refractive index dependence on wavelength for both the cladding tube and the core Ge-doped glass used in the core are shown

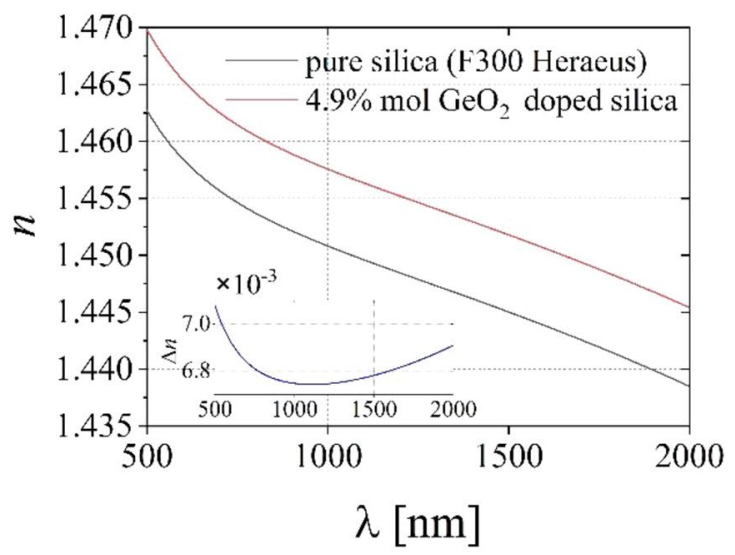

fiber and their refractive index difference. c SEM picture of the developed nGRIN fiber with parabolic core. The lighter areas indicate a distribution of the $\mathrm{GeO} 2$-doped silica rods, while the darker ones correspond to fused silica glass (Anuszkiewicz et al. 2018)

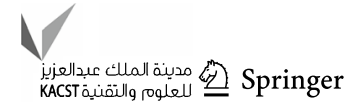


in Fig. 1b. The data for purse fused silica was taken from the vendors catalogs (Heraeus). Refractive index of the Gedoped silica was calculated using the Sellmeier dispersion equation (Fleming 1984):

$n^{2}(\lambda)-1=\sum_{i=1}^{3} \frac{\left[\mathrm{BS}_{i}+X\left(\mathrm{BG}_{i}-\mathrm{BS}_{i}\right)\right] \lambda^{2}}{\lambda^{2}-\left[\mathrm{CS}_{i}+X\left(\mathrm{CG}_{i}-\mathrm{CS}_{i}\right)\right]^{2}}$,

where $\lambda$ is the wavelength in vacuum, and $\mathrm{BS}_{i}, \mathrm{CS}$, and $\mathrm{BG}_{i}$, $\mathrm{CG}_{i}$ are the Sellmeier coefficients for the $\mathrm{SiO}_{2-}$ and $\mathrm{GeO} 2-$ doped glasses, respectively (Malitson 1965). The value of $X$ is the $\mathrm{GeO}_{2}$ concentration in $\% \mathrm{~mol}$ (here $X=4.9 \mathrm{~mol} \%$ ). The internal fiber structure was calculated based on the Maxwell-Garnett effective medium approach to obtain the parabolic profile of the effective refractive index distribution in the core. A dedicated simulated annealing algorithm was used to determine final distribution of nanorods in the core. Simulated annealing is a Monte Carlo method, in which the design procedure begins with a random material distribution and in each iteration, one random pixel is changed before a cost function is calculated (Kirkpatrick et al. 1983). The goal of the procedure is the minimization of the cost function. In our case, the cost function is defined as a difference between refraction index distribution in ideal parabolic gradient index profile and effective refractive index profile of nanostructured medium (Hudelist et al. 2009).

The fiber is developed using the standard stack-and-draw method, where a set of low and high refractive index rods form a preform with calculated internal core structure as shown in Fig. 1a. The outer fiber diameter was $135.8 \mu \mathrm{m}$ and the nanostructured core diameter was $7.15 \mu \mathrm{m}$. As shown in Fig. 1c, the internal structure of the designed layout of the core was well preserved during the fiber drawing process. Detailed description of design and fabrication process is given in a previous work (Anuszkiewicz et al. 2018).

\section{Characterization of fiber optical parameters}

Chromatic dispersion of the fiber of length $20.4 \mathrm{~cm}$ was measured using an unbalanced Mach-Zehnder interferometer. As the reference light source, we used a compact supercontinuum laser (LEUKOS) with spectral coverage spanning from 450 to $2400 \mathrm{~nm}$. Light was coupled into the fiber using a lens $\left(L_{1}\right)$ with focal length of $2 \mathrm{~mm}$. Outcoupling from the fiber was realized with a lens $\left(L_{2}\right)$ with $4.5 \mathrm{~mm}$ focal length. The interference fringes were registered with set of detectors: an optical spectrum analyzer (Yokogawa AQ6375B) operating from 1200 to $2400 \mathrm{~nm}$ and a compact Czerny-Turner spectrometer (Ocean Optic Red Tide USB650) for wavelength range of 350-1000 nm. The optical path difference (OPD) introduced by the fiber placed in the signal arm were measured as a function of the equalization wavelength. During calculation of fiber dispersion, we also took into account the small OPD induced by the optical elements introduced in the optical setup, knowing the dispersion and thickness characteristics of the lenses and filters. A scheme of the measurement setup is shown in Fig. 2.

\section{Generation of supercontinuum}

The nGRIN fiber was pumped with a $1550 \mathrm{~nm}$ modulated amplified laser diode. The diode has a peak power of $9.4 \mathrm{~kW}$ and deliver $1 \mathrm{~ns}$ pulses with $250 \mathrm{kHz}$ repetition rate. We used $\times 20$ microscope objective to couple input beam into nGRIN fiber. A $37 \%$ of coupling efficiency was achieved; however, the coupling system was not optimized. Average power injected in the fiber was $100 \mathrm{~mW}$, which correspond to $400 \mathrm{~nJ}$ pulse energy. The output beam was collected directly with optical spectrum analyzer (Yokogawa AQ6375B).

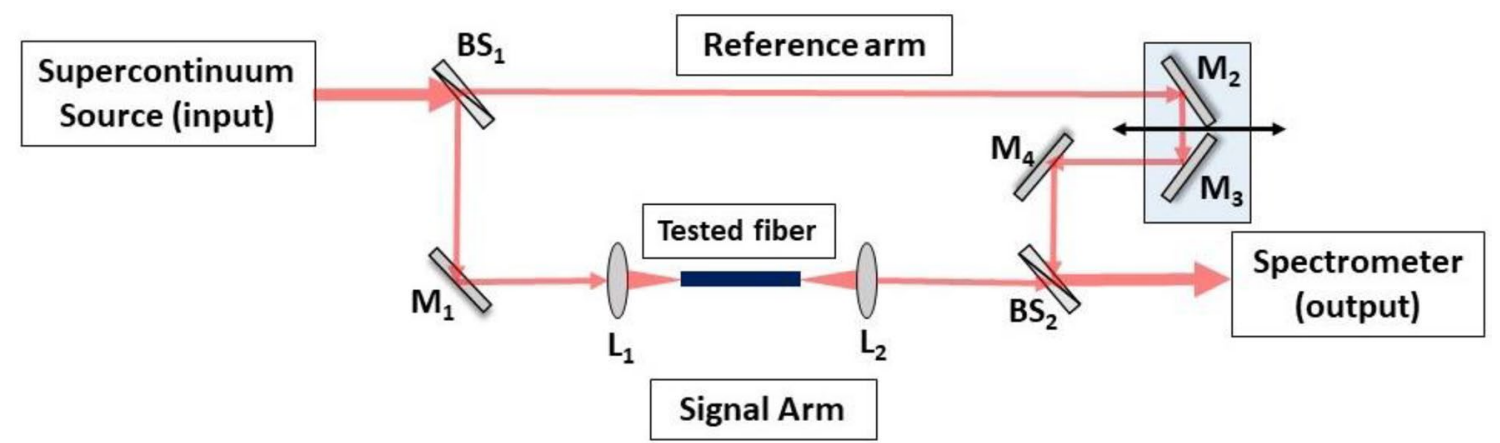

Fig. 2 Mach-Zehnder interferometer used for chromatic dispersion measurements 


\section{Results}

\section{Fiber characterization}

The nGRIN fiber labeled MS15B has monotonically increasing dispersion profile with relatively low, positive slope over the entire wavelength range over which spectral broadening occurs in experiments conducted in this work, Fig. 3. The zero-dispersion wavelength (ZDW) is located at $1370 \mathrm{~nm}$. This enables use of standard nanosecond pulse lasers to pump it efficiently at wavelengths, where the fiber has anomalous dispersion, which facilitates efficient supercontinuum generation. The maximum wavelength range covered in our measurements is $2400 \mathrm{~nm}$, where the fiber's dispersion reaches $60 \mathrm{ps} / \mathrm{nm} / \mathrm{km}$. At the short wavelength range below

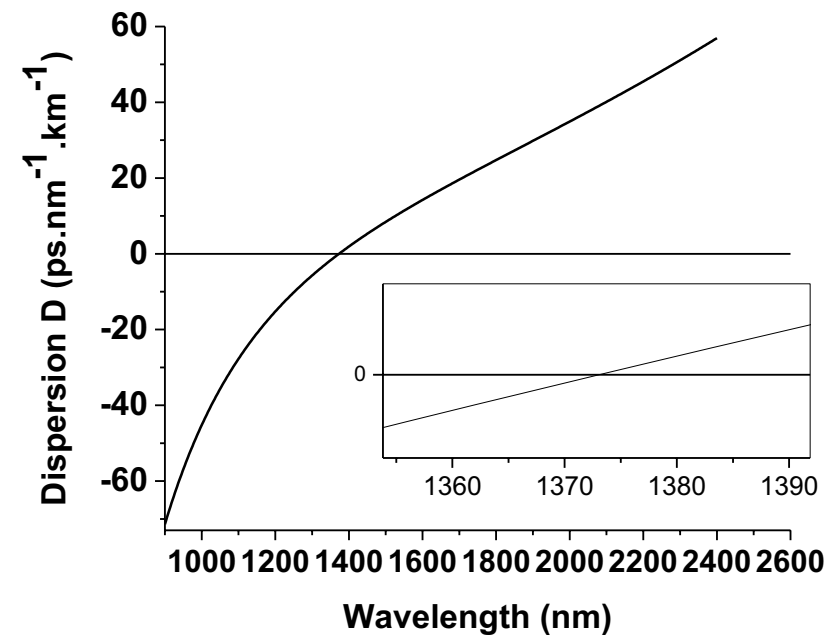

Fig. 3 Measurements of chromatic dispersion $(D)$ for the fundamental mode, from 900 to $2400 \mathrm{~nm}$ for MS15B3 fiber

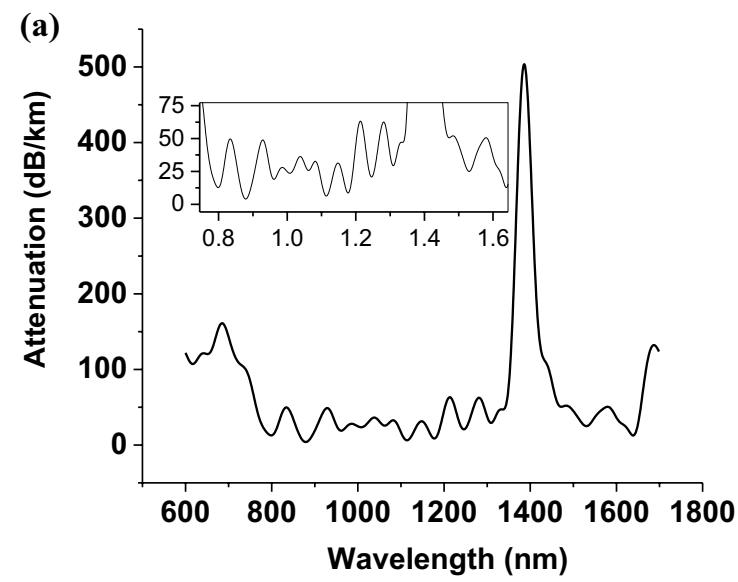

$1370 \mathrm{~nm}$, the dispersion profile reaches $-70 \mathrm{ps} / \mathrm{nm} / \mathrm{km}$ at a wavelength of $900 \mathrm{~nm}$. Such a dispersion profile is typical for silica single-mode fibers.

The confinement losses of the nGRIN fiber was measured using cut-back method in range $600-1700 \mathrm{~nm}$ starting with almost a $20 \mathrm{~m}$ long nGRIN fiber sample. Five consecutive cut-backs have been made down to $1.5 \mathrm{~m}$, (Anuszkiewicz et al. 2018). Attenuation of the whole developed nGRIN fiber series in this wavelength range remains below $50 \mathrm{~dB} /$ $\mathrm{km}$, besides the characteristic peak around $1400 \mathrm{~nm}$ due to the water content in the fiber structure, where the losses reach $500 \mathrm{~dB} / \mathrm{km}$, as shown in Fig. 4a. Figure $4 \mathrm{~b}$ contains spectrum recorded at the output of the starting length of the fiber sample $(19.5 \mathrm{~m})$ and the shortest sample (following five cut-backs) in the measurement. This part of experiment confirms that no additional source of pollution were introduced during the stacking of the preform. High level of the water peak is related to quality of silica rods used for fiber assembly and research-grade level of preform assembly conditions, where water level in the assembly cabinet was not reduced. A presence of water peak may influence on supercontinuum generation spectral range. The confinement losses of the fiber are not related fundamentally with stack-and-draw method and they can be significantly reduced when high-quality glass components are used to preform assembly in clean-room conditions, similarly to high-quality silica-based photonic crystal fibers previously reported (Nielsen et al. 2004).

\section{Modeling and measurements of supercontinuum generation in the nGRIN fiber}

The nonlinear propagation was modeled using general nonlinear Schrodinger equation (GNLSE). In nanosecond pulse pumping conditions, the primary nonlinear

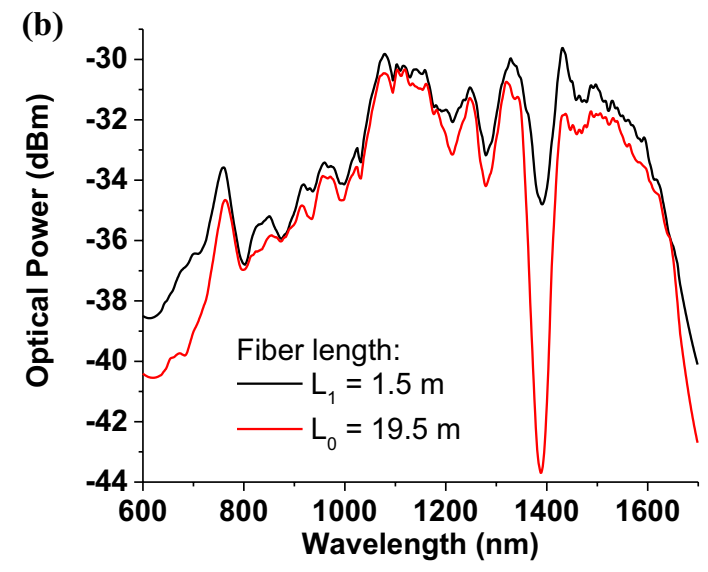

Fig. 4 a Attenuation of MS15B series measured by the cut-back method over wavelength range from 600 to $1700 \mathrm{~nm}$, b reference spectrum at the output of the fiber for the longest (starting) and the shortest (final) length of the fiber sample in the measurement 
phenomenon responsible for dramatic spectral broadening of the input pulse is modulation instability (MI) (Demircan and Bandelow 2005; Dudley et al. 2006, 2009). While numerical simulation of nonlinear propagation of nanosecond pulses would not be feasible to run on a standard personal computer, similar dynamics are observed under tens of picosecond pump pulses and such an approach was taken in this work. In our simulations, we input a Gaussian pulse with a temporal FWHM of 12 ps. Fiber length assumed in modeling was equal to $33.4 \mathrm{~cm}$ used in the following experiments. The width of temporal window grid of the simulation was $100 \mathrm{ps}$. The input peak power assumed in the simulation was $33.3 \mathrm{~kW}$, which corresponded with peak power of the laser in the experiment. The pump assumed in the modeling was operating at a wavelength of $1550 \mathrm{~nm}$ with a repetition rate of $250 \mathrm{kHz}$. At this wavelength, the fiber had anomalous dispersion of $D=+11.4 \mathrm{ps} / \mathrm{nm} / \mathrm{km}$. Frequency-dependent loss has been included in the simulations, taking into account the background loss level of $14.5 \mathrm{~dB} / \mathrm{km}$, a $500 \mathrm{~dB} / \mathrm{km}$ water peak centered around the wavelength of $1400 \mathrm{~nm}$ and a gradually rising attenuation towards the long-wavelength cut-off modeled after silica single-mode fibers. Fixed value of $55 \mu \mathrm{m}^{2}$ was used for the effective mode area in our modeling (Anuszkiewicz et al. 2018). For estimation of the nonlinear refractive index $n_{2}$ of this graded-index profile fiber, we used an approximate formula developed and established for a series of different Ge-doped silica single-mode fibers by Kato et al. (Kato et al. 1995). It has been proposed there to include parameters like the maximum (Ge-doped silica) and minimum (pure silica) refractive index values, as well as effective mode areas and radial distributions of the mode field intensity. For a given dopant concentration (in case of our fiber $\mathrm{Ge}$ ), the nonlinear refractive index can be approximated as $n_{2}=(2.76+0.0947 X) 10^{-20} \mathrm{~m}^{2} / \mathrm{W}$, where $X=4.9 \mathrm{~mol} \%$ stands for $\mathrm{GeO}_{2}$ concentration in the high index silica glass rods used for the nanostructured core preform stacking. The nonlinear refractive index values obtained for our fiber using this approximation is $3.2 \times 10^{-20} \mathrm{~m}^{2} / \mathrm{W}$. Finally, to take into account MI as the primary supercontinuum driving mechanism under long pulse pumping, we included a one photon with random phase in each numerical bin of the time window (onephoton-per-mode noise). A total of 20 simulations have been performed and averaged to obtain the final result. We performed several test simulation runs to verify, that after around 20 simulations, the averaged supercontinuum spectrum did not change significantly.

The time grid used in the simulation has to be wide enough to accommodate entire initial pulse, as well as entire output pulse, which is evidenced in Fig. 5 showing temporal evolution of the pump pulse from input of the fiber to its output.

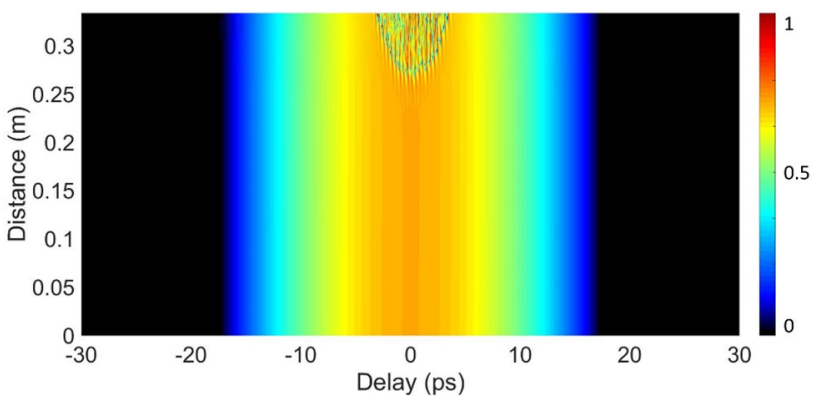

Fig. 5 Temporal profile evolution of the simulated supercontinuum (picosecond) pulse along the $33.4 \mathrm{~cm}$ length of the fiber, colorbar: normalized intensity in linear scale (a.u.)

The implemented GNLSE model takes into account the effects of chromatic dispersion, Raman scattering and the nonlinear response of fused silica with germanium doping. Spectral broadening in the 'long pulse regime' leads to pulse breakup in the time domain due to the fluctuations in the noise-induced modulation instability. Further spectral broadening in the fiber is due to the high number of solitons present after wave-breaking, as can be seen in Fig. 5. With the above parameters, the numerically obtained spectral broadening reached to $2300 \mathrm{~nm}$ at $100 \mathrm{~mW}$ input average power, which agrees with the results obtained experimentally and discussed below (Fig. 6). The consequence of using picosecond or longer pump pulses is decoherence of the input pulse along propagation in the fiber, which is the cost of rapid and broadband spectral broadening of the pulse spectrum (Demircan and Bandelow 2005). We verified the dominant role of MI in spectral broadening by running the nonlinear propagation simulations with one-photon-per-mode noise included and taken out of the model. We note that upon excluding of the noise term from the simulation, spectral broadening is limited to a Raman scattering cascade and no continuous spectrum is observed, Fig. $6 \mathrm{~b}$. In absence of noise, only Raman scattering peaks are observed.

In the experiment, we used fibers with different lengths, between around a $12 \mathrm{~cm}$ to over $1 \mathrm{~m}$. No significant spectral broadening could be obtained in samples longer than around $30 \mathrm{~cm}$. At shorter sample lengths, excessive unconverted pump was recorded. While the $33.4 \mathrm{~cm}$ fiber sample was ultimately used out of convenience, it is reasonably close to optimal for this case. The factors, which could be related to this include high attenuation of the fiber around $1400 \mathrm{~nm}$ due to $\mathrm{OH}$ absorption and non-optimal spectral separation between the ZDW at $1370 \mathrm{~nm}$ and the $1560 \mathrm{~nm}$ pump wavelength. We observed spectral broadening steadily increasing with the input energy from the pump laser diode. At $100 \mathrm{~mW}$ of average power, which corresponded to pulse energy of $400 \mathrm{~nJ}$, a spectrum spanning 1400-2300 nm was recorded, as shown in Fig. 7. As a result of the large separation of the pump wavelength from the ZDW and very poor 

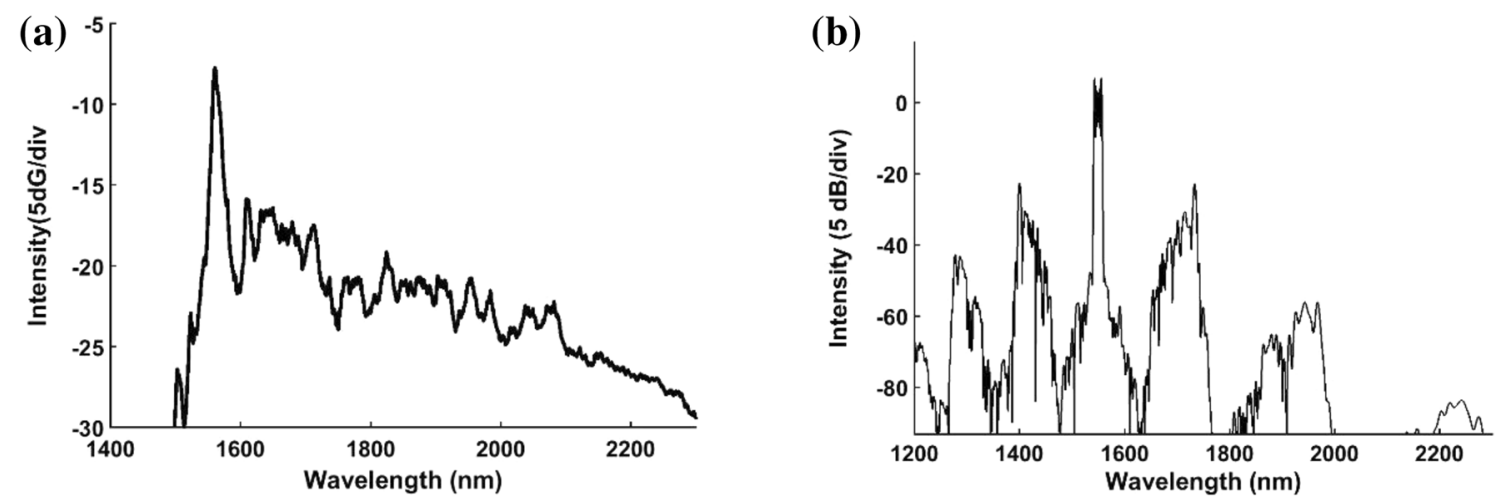

Fig. 6 a Numerical simulation showing supercontinuum generation due to noise-driven MI. b Raman scattering of cascading solitons in the absence of noise seeds in the simulation

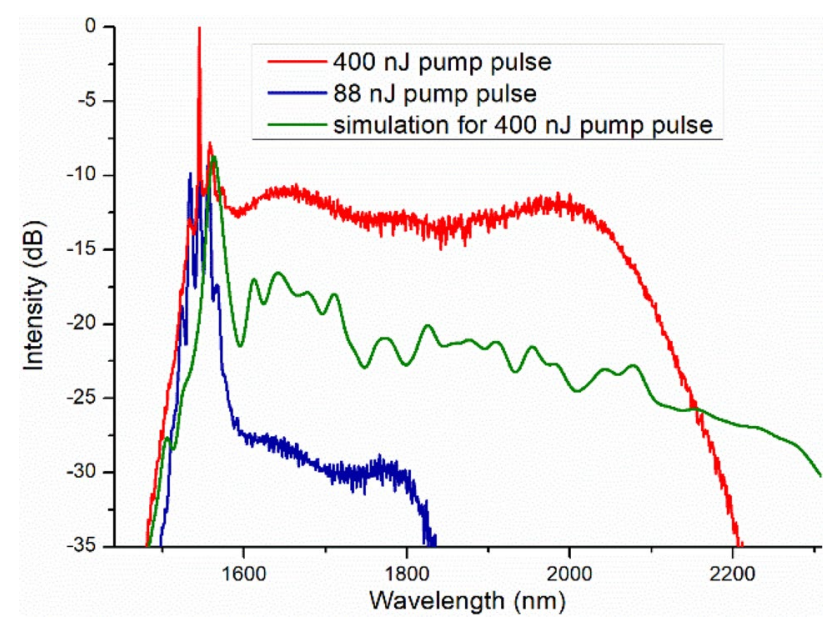

Fig. 7 Supercontinuum generated in MS15B3 nGRIN fiber using a $1550 \mathrm{~nm}$ laser diode $1 \mathrm{~ns}$ pulses and simulation results

efficiency of dispersive wave generation, as well as large attenuation of fiber around $1400 \mathrm{~nm}$ due to $\mathrm{OH}$ absorption, the short wavelength part of the supercontinuum is missing, as compared to e.g., state of the art visible to near-infrared commercial supercontinuum lasers (Leukos; NKTPhotonics). The red-shifted spectrum almost matches the commercial counterparts with its maximum wavelength reach. Generated supercontinuum is remarkably flat, which is a trait of modulation instability-driven broadening process (Bandelow and Demircan 2005). Its limited spectral width of around $800 \mathrm{~nm}$ (between 1500 and $2300 \mathrm{~nm}$, which is a sought-after spectral range in tissue OCT) has the important advantage of significantly higher spectral power density compared to the commercial supercontinuum devices covering in excess of two octaves under the same pump power. The numerically generated supercontinuum matches the experimental result with rather limited accuracy, which we assign to limited accuracy in parametrizing the nonlinear properties of the fiber, i.e. its dispersion of nonlinearity (frequency dependence of the effective mode area). In the simulation, flat fiber nonlinearity was assumed corresponding to a fixed value of nonlinear coefficient. We note, that in the physical fiber used in the experiment, expansion of the mode area with wavelength would decrease nonlinearity and limit extending of spectrum. This discrepancy between the experiment and simulation motivates detailed study of effective mode area dependence on wavelength in this series of fibers, which would be subject of a future study. Considering that the used fiber is short and robust (silica, no airholes, compatible with high pulse energy pumping from a laser diode), it could be an interesting alternative for cheap, high-brightness, hand-held light sources for medical OCT applications. Importantly, the nGRIN fiber concept has been discussed in context of broad-bandwidth chromatic dispersion engineering (Michalik et al. 2019; Siwicki et al. 2017), which could enable improved designs of fibers for enhanced spectral coverage of MI-driven supercontinuum in a small foot-print package. Furthermore, the nGRIN concept enabling mode profile engineering would be advantageous for cascaded supercontinuum setups (Israelsen et al. 2019). This is because the nGRIN fiber does not have airholes and the effective mode area can be matched at the fiber design level to the preceding and following fiber in the cascade.

\section{Conclusions}

We have used a nanostructured core optical fiber for the first time in a nonlinear fiber optics application, specifically in a supercontinuum generation experiment. The obtained supercontinuum spectrum covered wavelength range from 1400 to $2300 \mathrm{~nm}$ and can be considered typical for the chromatic dispersion profile measured for the used fiber, and for the $1550 \mathrm{~nm}$, nanosecond pulse pump laser used in the experiment. The main finding of our work is revealing that

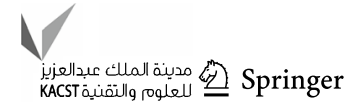


the internal nanostructure of the fiber core does not introduce any additional nonlinear effects related to this discrete core structure and this nanostructured fiber can be treated as an effective optical medium. The single-mode nGRIN fiber used in our experiments was not optimized for hyperspectral broadening in terms of the zero-dispersion wavelength, but nanostructuring allows for large modification of the dispersion profile through modification of the all-solid glass core, which we have demonstrated previously using numerical simulations for designing of normal dispersion profiles or shifting of the zero-dispersion wavelength (Siwicki et al. 2017; Michalik et al. 2019). Thus, the nanostructured core optical fibers have been shown as an alternative in chromatic dispersion engineering in fibers for nonlinear optics applications. The results reported in this work complement the state-of-the-art results available in literature (Demircan and Bandelow 2005; Dudley et al. 2006). We confirmed that the developed nGRIN fiber can withstand high-energy laser pump pulses without laser-induced damage, which we relate to its all-solid glass structure made of silica. Our work opens up new applications in power scaling of supercontinuum lasers using large-mode area fibers and new supercontinuum laser concepts using free-form nanostructured core fibers.

Funding European Training Network H2020-MSCA-ITN-2016 Grant no. 722380, SUPUVIR: Supercontinuum broadband light sources covering UV to IR applications; Project TEAM TECH/2016-1/1 (POIR.04.04.00-1C74/16) operated within the Foundation for Polish Science Team Programme co-financed by the European Regional Development Fund under Smart Growth Operational Programme (SG OP), Priority Axis IV.

\section{Compliance with ethical standards}

Conflict of interest The authors declare that they have no conflict of interest.

Open Access This article is licensed under a Creative Commons Attribution 4.0 International License, which permits use, sharing, adaptation, distribution and reproduction in any medium or format, as long as you give appropriate credit to the original author(s) and the source, provide a link to the Creative Commons licence, and indicate if changes were made. The images or other third party material in this article are included in the article's Creative Commons licence, unless indicated otherwise in a credit line to the material. If material is not included in the article's Creative Commons licence and your intended use is not permitted by statutory regulation or exceeds the permitted use, you will need to obtain permission directly from the copyright holder. To view a copy of this licence, visit http://creativecommons.org/licenses/by/4.0/.

\section{References}

Alfano RR, Shapiro SL (1970a) Emission in the region 4000-7000 $\AA$ via four-photon coupling in glass. Phys Rev Lett 24:584-587. https://doi.org/10.1103/PhysRevLett.24.584

Alfano RR, Shapiro SL (1970b) Observation of self-phase modulation and small-scale filaments in crystals and glasses. Phys Rev Lett 24:592-594. https://doi.org/10.1103/PhysRevLett.24.592

Anuszkiewicz A, Bidus M, Filipkowski A et al (2019) Experimental analysis of axial stress distribution in nanostructured core fused silica fibers. Opt Mater Express 9:4370. https://doi.org/10.1364/ OME.9.004370

Anuszkiewicz A, Kasztelanic R, Filipkowski A et al (2018) Fused silica optical fibers with graded index nanostructured core. Sci Rep 8:1-13. https://doi.org/10.1038/s41598-018-30284-1

Bandelow U, Demircan A (2005) Supercontinuum generation by the modulation instability. In: Frontiers in optics. OSA, Washington, DC, p FWI5. https://doi.org/10.1364/FIO.2005.FWI5

Chen H, Chen S, Wang J et al (2011) $35 \mathrm{~W}$ high power all fiber supercontinuum generation in PCF with picosecond MOPA laser. Opt Commun 284:5484-5487. https://doi.org/10.1016/J.OPTCO M.2011.08.024

Demircan A, Bandelow U (2005) Supercontinuum generation by the modulation instability. Opt Commun 244:181-185. https://doi. org/10.1016/J.OPTCOM.2004.09.049

Dudley JM, Coen S (2002) Coherence properties of supercontinuum spectra generated in photonic crystal and tapered optical fibers. Opt Lett 27:1180. https://doi.org/10.1364/OL.27.001180

Dudley JM, Genty G, Coen S (2006) Supercontinuum generation in photonic crystal fiber. Rev Mod Phys 78:1135-1184. https://doi. org/10.1103/RevModPhys.78.1135

Dudley JM, Genty G, Dias F et al (2009) Modulation instability, Akhmediev breathers and continuous wave supercontinuum generation. Opt Express 17:21497. https://doi.org/10.1364/ OE.17.021497

Feng X, Monro T, Petropoulos P et al (2003) Solid microstructured optical fiber. Opt Express 11:2225. https://doi.org/10.1364/ OE.11.002225

Fleming JW (1984) Dispersion in $\mathrm{GeO}_{2}-\mathrm{SiO}_{2}$ glasses. Appl Opt 23:4486. https://doi.org/10.1364/AO.23.004486

Franczyk M, Stawicki K, Lisowska J et al (2018) Numerical studies on large-mode area fibers with nanostructured core for fiber lasers. J Light Technol. https://doi.org/10.1109/JLT.2018.2873164

Franczyk M, Stepien R, Filipkowski A et al (2019) Nanostructured core active fiber based on ytterbium doped phosphate glass. J Light Technol 8724:1-1. https://doi.org/10.1109/jlt.2019.2941664

Fujimoto JG (2003) Optical coherence tomography for ultrahigh resolution in vivo imaging. Nat Biotechnol 21:1361-1367. https://doi. org/10.1038/nbt892

Genier E, Bowen P, Sylvestre T et al (2019) Amplitude noise and coherence degradation of femtosecond supercontinuum generation in all-normal-dispersion fibers. J Opt Soc Am B 36:A161. https://doi.org/10.1364/JOSAB.36.00A161

Hudelist F, Buczynski R, Waddie AJ, Taghizadeh MR (2009) Design and fabrication of nano-structured gradient index microlenses. Opt Express. https://doi.org/10.1364/oe.17.003255

Hudson DD, Antipov S, Li L et al (2017) Toward all-fiber supercontinuum spanning the mid-infrared. Optica 4:1163. https://doi. org/10.1364/OPTICA.4.001163

Israelsen NM, Petersen CR, Barh A et al (2019) Real-time high-resolution mid-infrared optical coherence tomography. Light Sci Appl 8:11. https://doi.org/10.1038/s41377-019-0122-5

Jiang X, Joly NY, Finger MA et al (2015) Deep-ultraviolet to midinfrared supercontinuum generated in solid-core ZBLAN photonic 
crystal fibre. Nat Photonics 9:133-139. https://doi.org/10.1038/ nphoton.2014.320

Jones DJ, Diddams SA, Ranka JK et al (2000) carrier-envelope phase control of femtosecond mode-locked lasers and direct optical frequency synthesis. Science 288:635-639. https://doi.org/10.1126/ science.288.5466.635

Kasztelanic R, Filipkowski A, Anuszkiewicz A et al (2018) Integrating free-form nanostructured grin microlenses with single-mode fibers for optofluidic systems. Sci Rep 8:1-12. https://doi.org/10.1038/ s41598-018-23464-6

Kato T, Suetsugu Y, Nishimura M (1995) Estimation of nonlinear refractive index in various silica-based glasses for optical fibers. Opt Lett 20:2279. https://doi.org/10.1364/ol.20.002279

Kirkpatrick S, Gelatt CD, Vecchi MP (1983) Optimization by simulated annealing. Science 220:671-680. https://doi.org/10.1126/ science.220.4598.671

Klimczak M, Siwicki B, Zhou B et al (2016) Coherent supercontinuum bandwidth limitations under femtosecond pumping at $2 \mu \mathrm{m}$ in all-solid soft glass photonic crystal fibers. Opt Express 24:29406. https://doi.org/10.1364/OE.24.029406

Klimczak M, Siwicki B, Heidt A, Buczyński R (2017) Coherent supercontinuum generation in soft glass photonic crystal fibers. Photonics Res 5:710. https://doi.org/10.1364/PRJ.5.000710

Leukos (2019) Industrial supercontinuum lasers. Leukos. https://www. leukos-systems.com/our-products/supercontinuum-lasers/indus trial. Accessed 21 Dec 2019

Liu L, Cheng T, Nagasaka K et al (2016) Coherent mid-infrared supercontinuum generation in all-solid chalcogenide microstructured fibers with all-normal dispersion. Opt Lett 41:392. https://doi. org/10.1364/OL.41.000392

Malitson IH (1965) Interspecimen comparison of the refractive index of fused silica. J Opt Soc Am 55:1205. https://doi.org/10.1364/ JOSA.55.001205

Michalik D, Stefaniuk T, Buczyński R (2019) Dispersion management in hybrid optical fibers. J Lightwave Technol. https://doi. org/10.1109/JLT.2019.2952250

Nicholson JW, Yan MF, Wisk P et al (2003) All-fiber, octave-spanning supercontinuum. Opt Lett 28:643. https://doi.org/10.1364/ OL.28.000643

Nielsen MD, Jacobsen C, Mortensen NA et al (2004) Low-loss photonic crystal fibers for transmission systems and their dispersion properties. Opt Express 12:1372. https://doi.org/10.1364/ OPEX.12.001372

Oh KPUC (2012) Silica optical fiber technology for devices and components: design, fabrication, and international standards. WileyVCH Verlag GmbH \& Co. KGaA, Hoboken

Petersen CR, Moselund PM, Petersen C et al (2016) Spectral-temporal composition matters when cascading supercontinua into the mid-infrared. Opt Express 24:749. https://doi.org/10.1364/ OE.24.000749

Ranka JK, Windeler RS, Stentz AJ (2000) Visible continuum generation in air-silica microstructure optical fibers with anomalous dispersion at $800 \mathrm{~nm}$. Opt Lett 25:25. https://doi.org/10.1364/ OL.25.000025

Shi K, Li P, Yin S, Liu Z (2004) Chromatic confocal microscopy using supercontinuum light. Opt Express 12:2096. https://doi org/10.1364/OPEX.12.002096

Siwicki B, Filipkowski A, Kasztelanic R et al (2017) Nanostructured graded-index core chalcogenide fiber with all-normal dispersiondesign and nonlinear simulations. Opt Express 25:12984. https:// doi.org/10.1364/OE.25.012984

Śmietana M, Koba M, Mikulic P, Bock WJ (2016) Towards refractive index sensitivity of long-period gratings at level of tens of $\mu \mathrm{m}$ per refractive index unit: fiber cladding etching and nano-coating deposition. Opt Express 24:11897. https://doi.org/10.1364/ OE.24.011897

Tarnowski K, Martynkien T, Mergo P et al (2019) Compact all-fiber source of coherent linearly polarized octave-spanning supercontinuum based on normal dispersion silica fiber. Sci Rep 9:1-8. https://doi.org/10.1038/s41598-019-48726-9

Wei D-P, Galstian TV, Smolnikov IV et al (2005) Spectral broadening of femtosecond pulses in a single-mode As-S glass fiber. Opt Express 13:2439. https://doi.org/10.1364/opex.13.002439

Wetzel B, Kues M, Roztocki P et al (2018) Customizing supercontinuum generation via on-chip adaptive temporal pulse-splitting. Nat Commun 9:4884. https://doi.org/10.1038/s41467-018-07141-w

Publisher's Note Springer Nature remains neutral with regard to jurisdictional claims in published maps and institutional affiliations. 